
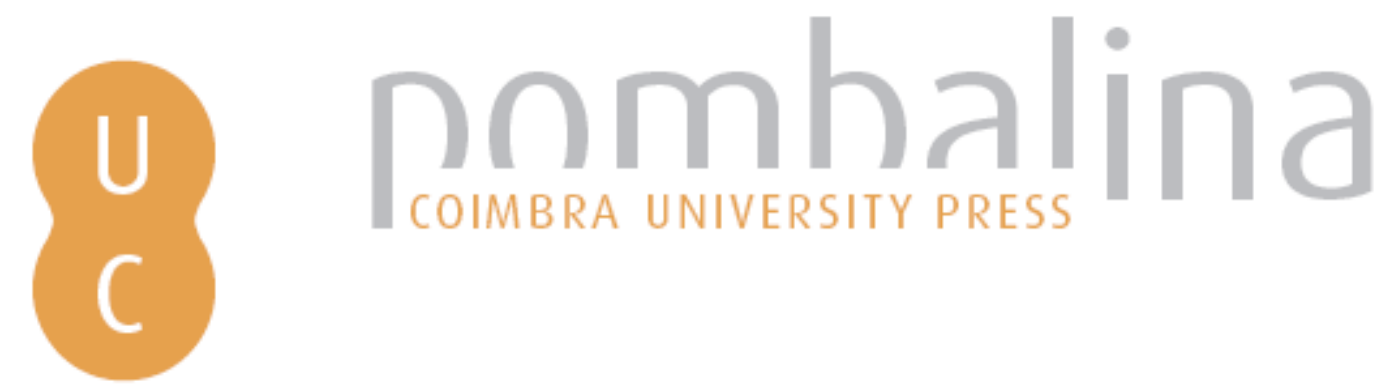

\title{
Nuevos espacios laborales para los titulados en información y documentación con especial referencia al mundo de la comunicación, la Administración Pública y la empresa
}

\author{
Autor(es): $\quad$ Chaín Navarro, Celia; Muñoz Cañavate, Antonio
}

Publicado por: Imprensa da Universidade de Coimbra

URL persistente:

URI:http://hdl.handle.net/10316.2/31961

DOI:

DOI:http://dx.doi.org/10.14195/978-989-26-0319-3_37

Accessed : $\quad$ 26-Apr-2023 13:42:21

A navegação consulta e descarregamento dos títulos inseridos nas Bibliotecas Digitais UC Digitalis, UC Pombalina e UC Impactum, pressupõem a aceitação plena e sem reservas dos Termos e Condições de Uso destas Bibliotecas Digitais, disponíveis em https://digitalis.uc.pt/pt-pt/termos.

Conforme exposto nos referidos Termos e Condições de Uso, o descarregamento de títulos de acesso restrito requer uma licença válida de autorização devendo o utilizador aceder ao(s) documento(s) a partir de um endereço de IP da instituição detentora da supramencionada licença.

Ao utilizador é apenas permitido o descarregamento para uso pessoal, pelo que o emprego do(s) título(s) descarregado(s) para outro fim, designadamente comercial, carece de autorização do respetivo autor ou editor da obra.

Na medida em que todas as obras da UC Digitalis se encontram protegidas pelo Código do Direito de Autor e Direitos Conexos e demais legislação aplicável, toda a cópia, parcial ou total, deste documento, nos casos em que é legalmente admitida, deverá conter ou fazer-se acompanhar por este aviso.

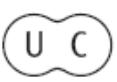


Maria Manuel Borges

Elias Sanz Casado

Coordenação

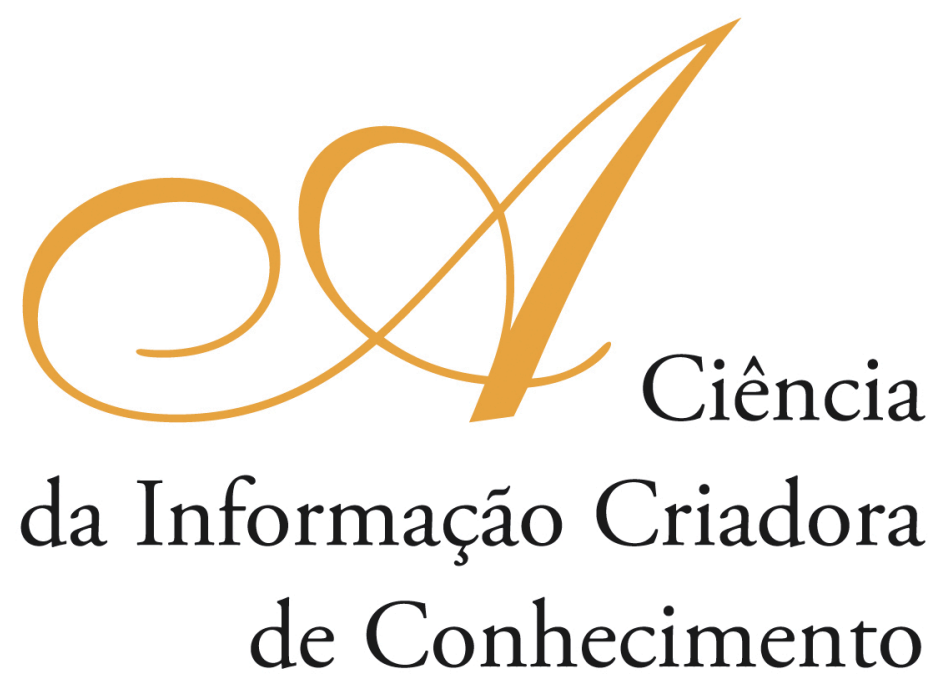

Vol. I

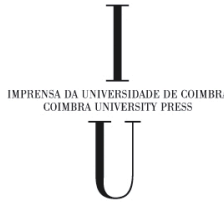

- COIMBRA 2009 
NuEVOS ESPACIOS LABORALES PARA LOS TITULADOS

EN INFORMACIÓN Y DOCUMENTACIÓN CON ESPECIAL REFERENCIA AL MUNDO DE LA comunicación, la Administración Pública y la empresa

\author{
Celia Chaín Navarro \\ Universidad de Murcia (Espanha) \\ Antonio Muñoz Cañavate \\ Universidad de Extremadura (Espanha)
}

\title{
Resumen
}

Durante los últimos años se ha producido en el mundo de la Información y la Documentación un proceso de cambio que ha permitido vislumbrar nuevos espacios laborales, docentes y de investigación, inexistentes hace dos décadas.

Este proceso de cambio ha venido derivado de la aceptación del recurso información como un activo más en las organizaciones. En este contexto, y dentro del ciclo integral de la gestión de la información, los titulados universitarios en Información y Documentación se convierten, o deberían convertirse, en elementos esenciales del control y gestión de la información a todos los niveles, y en todo tipo de organizaciones públicas y privadas.

Distintos autores han ofrecido explicaciones acerca de la transformación que ha experimentado la sociedad actual, y que entronca de manera directa y explícita con el papel de la Universidad en su entorno. Entre las razones se encuentran el importante desarrollo científico y técnico, o el enorme crecimiento económico, a pesar de las crisis económicas coyunturales. Todo ello ha derivado en una sociedad muy compleja, que requiere demandas de empleo muy diversificadas y especializadas que deben ser cubiertas a través de los diversos procesos formativos, sean dentro o fuera de la Universidad.

En este trabajo los autores presentan un catálogo de nuevas oportunidades potenciales que se han abierto a los titulados en Biblioteconomía y Documentación en distintos ámbitos, como el mundo de la comunicación y la publicidad, la Administración Pública y la empresa.

\section{Introducción}

La formación universitaria española en Información y Documentación, o si se quiere en Biblioteconomía y Documentación -utilizando la terminología más comúnmente conocida en España- se remonta a los años ochenta del siglo XX. Si bien cuando se estudia la historia de la Documentación espańola siempre se hace referencia a determinados hitos anteriores que, de una u otra forma, han sido notables iniciativas para la formación de los profesionales de bibliotecas y archivos en España. Nos referimos, por ejemplo, a la Escuela de Diplomática creada en 1856, y que funcionó hasta 1900, o la Escuela Superior de Bibliotecarias de Barcelona, que comenzó a funcionar en 1915 .

Sin embargo, parece que queda fuera de toda duda que la madurez en la formación de los profesionales llega en Espańa con la implantación de la Diplomatura 
de Biblioteconomía y Documentación, y posteriormente de la Licenciatura en Documentación. Dos títulos que han iniciado un proceso de desaparición de todas las universidades en las que se encuentran implantadas, ya que la adaptación al Espacio Europeo de Educación Superior (Plan Bolonia) ha dado lugar a la creación de un nuevo título de grado denominado "Título de Grado en Información y Documentación".

Más de dos décadas de formación universitaria pueden dar para muchas reflexiones y sin duda para un arduo debate que defina qué se ha realizado, qué se ha conseguido y hacia dónde deben de dirigirse los estudios.

En los eventos espańoles (congresos, jornadas, cursos) que reúnen a los profesionales y académicos de la Información y Documentación en España, además de en los foros electrónicos como Iwetel, se ha abierto un debate, a veces agrio, que trata de analizar la realidad española, y las metas alcanzadas.

Los autores de este trabajo parten del convencimiento de que, desde el interior de la universidad, no ha habido el suficiente debate que haya permitido reflexionar acerca de las innumerables posibilidades tanto académicas (docentes y de investigación) como profesionales, que nos ha traído el paradigma de la Sociedad de la Información.

Los autores que firman este trabajo citan con frecuencia las palabras de dos expertos españoles en política universitaria, Francisco Michavila y Benjamín Calvo. Estos autores insisten en la transformación que ha experimentado la sociedad actual y que supone una posición que haga repensar el papel de las universidades, en tanto que esa Sociedad de la Información y el enorme desarrollo científico y tecnológico, han dado lugar a nuevas necesidades en la sociedad que antes no existían, con demandas muy diversificadas y empleo cambiante. Así, señalan "preciso es recordar la afirmación aparcada de una revista de difusión europea, según la cual la mitad de las profesiones que estarán vigentes dentro de veinticinco años son desconocidas". Y nos dicen: ¿Quién puede pensar que la Universidad tradicional, con sus sólidas enseñanzas, magistralmente impartidas por profesores que las aprendieron hace cuarenta años, puede dar una respuesta satisfactoria? (Michavila \& Calvo, 1998).

Hemos de tener en cuenta que ha sido la desregulación del mercado laboral el fenómeno que más incide en el dinamismo de los distintos perfiles profesionales, lo que quiere decir que a un mismo trabajo pueden acceder personas de distintas titulaciones. Por ejemplo, José María Peiró, director del Observatorio de Inserción Profesional y Asesoramiento Laboral de la Universidad de Valencia, nos señala que "hasta hace unos años todavia podia decirse que a una determinada carrera le correspondia un determinado trabajo. Ahora a un mismo puesto pueden acudir personas de distintas titulaciones, y una titulación puede llevar a puestos de trabajo que clásicamente no se percibian como propios" de tal manera que cuando se opta por una carrera no se emprende un camino estanco, sino que existe una amplia oferta en la que moverse ${ }^{1}$.

Estas reflexiones, junto con una mayor adaptación de los planes de estudio de las universidades a las nuevas realidades que se crean, son las que deben generar, en

${ }^{1}$ Diario El País, 28-9-2008, p. 3. 
opinión de los autores de este trabajo, un proceso de adaptación y cambio que apenas se vislumbra.

Muchas pueden ser las causas que han dado lugar a esa falta de reflexión, por ejemplo, la profesora española Purificación Moscoso, en un trabajo orientado a conocer el grado de implicación de la tecnología en la enseñanza universitaria en Documentación, señala que el perfil del profesorado espańol es de un marcado perfil humanístico (Moscoso, 2000), lo que quiere decir que durante varias décadas la enseñanza de la Documentación en España se ha nutrido, sobre todo, de docentes del campo de las humanidades que ha impedido un proceso interno de modernización. De esta manera, desde la propia universidad se ha establecido una visión restringida del mundo de la Información y la Documentación, lo cual ha sido una rémora que ha impedido profundizar en una perspectiva más amplia de los objetivos académicos y de la profesión.

El profesor García Marco (2008), señala, además, que en los debates en los últimos años también aparece la existencia de otros problemas de fondo, como son la automatización creciente frente a una enseńanza centrada en los procedimientos artesanales; la permanente crisis de identidad de la Ciencia de la Información —a la que no ayuda la pluralidad paradigmática y metodológica de un campo netamente interdisciplinar (McNicol, 2003) —; o la debilidad en recursos humanos y financieros respecto a otras profesiones y disciplinas; etc.

Si bien todos estos problemas no son exclusivos de la Biblioteconomía e Información y Documentación española, ya que son cuestiones comunes a una parte importante de las titulaciones europeas (Audunson, 2005) y de las estadounidenses y canadienses (Weech, 2007).

\section{Algunos datos de los estudios de Biblioteconomía y Documentación en España}

Las tablas 1 y 2 muestran los datos oficiales del Instituto Nacional de Estadística acerca de la evolución de los alumnos matriculados en los dos títulos existentes en España: la Diplomatura de Biblioteconomía y la Licenciatura en Documentación. Sobre la base 100 que representa el curso 1996/1997 se puede observar la evolución de los siguientes cursos, comparando los datos con el total de todas las titulaciones universitarias espańolas que imparten títulos de Diplomatura (tabla 1) y del total de todas las titulaciones universitarias españolas que imparten títulos de Licenciatura (tabla 2). Así, se compara la matrícula registrada en la Diplomatura de Biblioteconomía y Documentación con la matrícula registrada en todas los títulos universitarios de Diplomatura; y la matrícula registrada en la Licenciatura en Documentación con la matrícula registrada en todas los títulos universitarios de Licenciado.

Aquí se observa una caída importante del número de alumnos matriculados en Diplomatura de Biblioteconomía y Documentación desde el curso 2002/2003 que tiene consecuencias casi dramáticas en el curso 2006/2007; y un crecimiento de los alumnos matriculados en los estudios de Licenciatura en Documentación que tiene su punto álgido en el curso 2001/2002, y que comienza a caer a partir de esa fecha.

Sin embargo, es necesario reflejar en este trabajo que en España ha sido normal que a la Licenciatura en Documentación accediesen, además de alumnos procedentes 
de la Diplomatura de Biblioteconomía y Documentación, titulados de otras carreras universitarias en busca de un título de segundo ciclo como es la Licenciatura en Documentación. Así, titulados en Fisioterapia o Enfermería (entre otros títulos) han buscado ese objetivo, al margen de otros titulados que buscaban una especialización en Documentación.

Tabla 1 - Evolución de la matriculación en la Diplomatura.

\begin{tabular}{|l|c|c|c|c|c|c|c|c|c|c|c|}
\hline & $\begin{array}{c}1996 / \\
1997\end{array}$ & $\begin{array}{c}1997 / \\
1998\end{array}$ & $\begin{array}{c}1998 / \\
1999\end{array}$ & $\begin{array}{c}1999 / \\
2000\end{array}$ & $\begin{array}{c}2000 / \\
2001\end{array}$ & $\begin{array}{c}2001 / \\
2002\end{array}$ & $\begin{array}{c}2002 / \\
2003\end{array}$ & $\begin{array}{c}2003 / \\
2004\end{array}$ & $\begin{array}{c}2004 / \\
2005\end{array}$ & $\begin{array}{c}2005 / \\
2006\end{array}$ & $\begin{array}{c}2006 / \\
2007\end{array}$ \\
\hline $\begin{array}{l}\text { Diplomatura } \\
\text { de ByD }\end{array}$ & 100 & 96,45 & 108,54 & 110,63 & 107,79 & 107,65 & 97,34 & 90,96 & 78,01 & 67,46 & 59,52 \\
\hline $\begin{array}{l}\text { Todas las } \\
\text { Diplomaturas } \\
\text { espańolas }\end{array}$ & 100 & 102,65 & 105,76 & 107,61 & 106,56 & 104,68 & 103,95 & 103,59 & 103,50 & 105,28 & 106,93 \\
\hline
\end{tabular}

Fuente: INE

Tabla 2 - Evolución de la matriculación en la Licenciatura

\begin{tabular}{|l|c|c|c|c|c|c|c|c|c|c|c|}
\hline & $\begin{array}{c}1996 / \\
1997\end{array}$ & $\begin{array}{c}1997 / \\
1998\end{array}$ & $\begin{array}{c}1998 / \\
1999\end{array}$ & $\begin{array}{c}1999 / \\
2000\end{array}$ & $\begin{array}{c}2000 / \\
2001\end{array}$ & $\begin{array}{c}2001 / \\
2002\end{array}$ & $\begin{array}{c}2002 / \\
2003\end{array}$ & $\begin{array}{c}2003 / \\
2004\end{array}$ & $\begin{array}{c}2004 / \\
2005\end{array}$ & $\begin{array}{c}2005 / \\
2006\end{array}$ & $\begin{array}{c}2006 / \\
2007\end{array}$ \\
\hline $\begin{array}{l}\text { Licenciatura } \\
\text { Documentación }\end{array}$ & 100 & 133,12 & 177,49 & 303,43 & 358,52 & 422,29 & 410,50 & 407,61 & 389,39 & 350,38 & 291,21 \\
\hline $\begin{array}{l}\text { Todas las } \\
\text { Licenciaturas } \\
\text { espańolas }\end{array}$ & 100 & 101,21 & 99,91 & 98,44 & 94,52 & 91,42 & 87,99 & 86,09 & 82,26 & 80,56 & 78,48 \\
\hline
\end{tabular}

Fuente: INE

En una reseña para ThikEPI Emilio Delgado, en un documento titulado El ocaso de las enseñanzas universitarias de Documentación en España, en su reflexión acerca de la caída de los matriculados en España nos decía:

"La conclusión no puede ser más desalentadora: mientras que la Universidad española crece y remonta la crisis producida en años anteriores como consecuencia del bajón demográfico, la DByD se hunde estrepitosamente. El hecho de que la $\mathrm{DByD}$ se desvíe de una manera tan acusada de la tendencia general universitaria induce a pensar que las raíces del problema son endógenas y no pueden atribuirse a una supuesta situación global de retraimiento. ¿Qué hemos hecho para caer tan bajo? o para plantearlo de una manera más positiva ¿qué no hemos hecho para seguir la misma estela de la universidad espańola? Estas preguntas exigen una respuesta seria y reflexiva para intentar enderezar la situación”... Para señalar más adelante "Tras 25 años instalados en los campus universitarios, hemos sido incapaces, sobre todo los que trabajamos como docentes en las 16 universidades donde se imparte nuestras titulaciones, de convencer a nuestros colegas acerca de la utilidad de la Documentación para el aprendizaje y la investigación y su legitimidad como disciplina”. 
A continuación vamos a dar unas pinceladas acerca de tres perfiles que desarrollan nuevas posibilidades para los titulados en Documentación, en los ámbitos de la Inteligencia Competitiva, la Administración electrónica y la comunicación publicitaria.

\section{Inteligencia Competitiva y Vigilancia tecnológica.}

Este es un concepto que ha comenzado a tener una cierta relevancia en el mundo universitario, y que la SCIP, la Sociedad de Profesionales de la Inteligencia Competitiva (sociedad creada en Estados Unidos en 1986), define como el proceso de conocimiento y análisis del entorno competitivo enfocado a una toma eficaz de decisiones.

Este proceso no es nuevo en el mundo de la economía y los negocios, así, por ejemplo, en la Espańa de finales del siglo XVIII en el Correo Mercantil y sus Indias, publicación que inicia su andadura el 1 de octubre de 1792, su editor, Gallard, nos decía que los comerciantes que alcanzaban un mayor éxito en sus empresas eran aquellos que conocían lo que ocurría a su alrededor y, por ello, les recomendaba tener en cuenta:

"los temporales, las cosechas, el aumento y disminución de consumos, la abundancia o escasez de frutos en los pueblos, sus variaciones en gustos, sus diferentes tratos y alianzas, sus providencias económicas, sus empresas mercantiles, su progreso en las artes".

La elevada concentración de pequeñas y medianas empresas en España, tal y como se puede comprobar en la tabla 3 hace que el análisis de la estructura empresarial española, a partir del tamaño de la empresa, nos lleve a pensar que difícilmente se pueda implementar un único modelo de responsables de la Inteligencia Competitiva (IC) en España.

Y es que el contexto español está constituido, en su mayoría, por empresas sin asalariados, con algo más de la mitad del total (51\%) y el $43 \%$ está formada por empresas con menos de 10 trabajadores. Estas cifras sumadas nos indican que en torno al $95 \%$ de las organizaciones empresariales españolas o no tienen asalariados o tienen una plantilla muy escasa. Por otro lado el 5,79\% corresponde a empresas que tienen entre 10 y 200 trabajadores. Y si sumamos el número de las que se encuentran por encima de los 200 trabajadores, el porcentaje total de empresas se sitúa en Espańa en el $0,18 \%$ del total.

Ello hace pensar que mientras hay empresas que pueden permitirse disponer de personal dedicado a la IC, otras tendrían que depender de agentes intermediarios. La generalización de asociaciones empresariales de todo tipo, y de estructuras de apoyo como los institutos tecnológicos radicados a lo largo del país, permite definir una estructura con agentes de la IC que, o bien en las propias empresas o en estructuras intermedias, posibiliten la generalización de este tipo de profesionales ${ }^{2}$

\footnotetext{
${ }^{2}$ En 2009 se ha creado en Espańa la Asociación Española de Promoción de la Inteligencia Competitiva (Asepic) que tiene entre otros objetivos definir un modelo de expansión de la IC en este país. En Extremadura,
} 
Tabla 3 - Empresas por estrato de asalariados 2007

\begin{tabular}{|l|c|c|}
\hline & Totales & \\
\hline Sin asalariados & 1.706 .140 & $51,133 \%$ \\
\hline De 1 a 2 asalariados & 932.526 & $27,948 \%$ \\
\hline De 3 a 5 asalariados & 340.638 & $10,209 \%$ \\
\hline De 6 a 9 asalariados & 158.159 & $4,740 \%$ \\
\hline De 10 a 19 asalariados & 108.866 & $3,263 \%$ \\
\hline De 20 a 49 asalariados & 60.738 & $1,820 \%$ \\
\hline De 50 a 99 asalariados & 15.737 & $0,472 \%$ \\
\hline De 100 a 199 asalariados & 7.780 & $0,233 \%$ \\
\hline De 200 a 499 asalariados & 4.218 & $0,126 \%$ \\
\hline De 500 a 999 asalariados & 1.072 & $0,032 \%$ \\
\hline De 1000 a 4999 asalariados & 684 & $0,020 \%$ \\
\hline De 5000 o más asalariados & 99 & $0,003 \%$ \\
\hline Total & 3.336 .657 & $100,00 \%$ \\
\hline
\end{tabular}

Fuente: INE

Queremos destacar en esta comunicación los estudios realizados por la ADBS (L'association des professionnels de l'information et de la documentation) francesa, en un país particularmente preocupado por la inteligencia competitiva como una herramienta imprescindible para la toma de decisiones. En 2001 publicó su Référentiel des métierstypes des professionnels de l'information-documentation (que sustituye al de 1998) y que complementa al Euro-referencial I\&D: referencial de competencias de los profesionales europeos en información y documentación.

En este documento se distingue un catálogo de 19 oficios (métier-type) a los que adjuntan sus competencias profesionales. También dispone de un grupo de 49 empleos -son actividades dentro de las organizaciones. De los 19 oficios (métier-type) destacamos aquí los trabajos que consideramos de suma importancia para la orientación laboral de los titulados:

a) "documentalista archivero" (métier-type 9)

b) "Gestor de documentos de empresa" (métier-type 12)

c) "vigilante-documentalista" (métier-type 19)

Este último perfil es el que se encarga de la vigilancia de todo tipo de informaciones y pueden realizar su trabajo en el interior de una empresa o como gestores independientes. Su función, por tanto, es la de suministrar información a los directivos de una empresa sobre la evolución de su entorno para ayudar a una mejor toma de decisiones.

la Administración regional en el contexto de su programa Innoveex destinado a potenciar la innovación en el tejido empresarial extremeño va a desarrollar en 2009 una estrategia específica de Inteligencia Competitiva. Así, la vicepresidenta económica de la Junta de Extremadura señaló en una jornada el 12 de marzo de 2009, que dentro de Innoveex se está desarrollando un sistema de vigilancia estratégica con el objetivo fundamental de introducir el concepto Inteligencia Competitiva en el tejido empresarial extremeńo. 
Finalmente, en el contexto de los esfuerzos franceses, en septiembre de 2006 siete organismos $^{3}$ del país vecino establecieron un listado de las funciones y competencias de la Inteligencia Económica ${ }^{4}$ formado por personas dedicadas a estas tareas a tiempo total o parcial:

a) Formador en Inteligencia Económica dentro de distintos niveles educativos.

b) Vigilante (dedicado a la búsqueda y difusión información escrita u oral).

c) Analista (que valida, interpreta y analiza la información).

d) Auditor en inteligencia económica (para implantar o reorganizar un sistema de inteligencia económica).

e) Consultor en inteligencia económica.

f) Editor de programas de inteligencia económica. Trabajos dedicados a recolectar y suministrar información útil a los vigilantes y analistas.

g) Directivo o responsable de inteligencia económica.

h) Delegado general de inteligencia económica (se diferencia del anterior en que realiza sus funciones en un territorio, una Administración o un grupo de organizaciones).

i) Lobby. Conduce las acciones para influir en los dominios de la inteligencia económica.

\section{El documentalista y la Administración electrónica}

En segundo ámbito de enorme potencial para el mundo de los profesionales y académicos de la Información y Documentación es la gestión de la información en las Administraciones Públicas que con las herramientas tecnológicas necesarias ha configurado un nuevo campo como es el de la Administración electrónica. Este nuevo ámbito se puede analizar desde el punto de vista de la gestión interna, lo que denominaríamos procesos back-office, y otro en la necesidad de estructurar mejor los flujos informativos a los ciudadanos y empresas y de mejora del servicio hacia su entorno, definiría los procesos front-office.

La tabla 4 nos presenta la distinta configuración de las Administraciones Públicas españolas que por su complejidad, derivada de la España de las autonomías, mantiene un elevado número de organismos y, por tanto, de empleo potencial.

${ }^{3}$ Assemblée des Chambres Françaises de Commerce et d'Industrie (ACFCI); Association des professionnels de l'information et de la documentation (ADBS); Agencia Nacional de Empleo (ANPE); Fédération des Professionnels de l'Intelligence Economique (FEPIE); Haut responsable en charge de l'intelligence économique (HRIE); Institut Français de l'Intelligence Economique (IFIE); Association Française pour la Promotion de l'Intelligence Economique et Concurrentielle (SCIP France).

4 Aunque no existe una terminología homogénea en todo el mundo para definir la Inteligencia competitiva, y, por ejemplo, los franceses hablan de inteligencia económica y competitiva, para los autores de este trabajo no hay diferencia, ni en el término, ni en el concepto. Las únicas diferencias las marca el tipo de información que se captura. 
Tabla 4 - La Administración Pública española y su ámbito territorial

\begin{tabular}{|l|l|l|}
\hline Territorio & Administración & Unidades \\
\hline \multirow{3}{*}{ España } & Administración General del Estado & $\begin{array}{l}1 \text { Gobierno central con } 16 \\
\text { ministerios y } \\
139 \text { organismos autónomos }\end{array}$ \\
\hline Comunidad Autónoma & Administración regional & $\begin{array}{l}17 \text { gobiernos regionales y } \\
\text { dos ciudades autónomas }\end{array}$ \\
\hline \multirow{3}{*}{ Provincias e Islas } & Administración local: diputaciones & 41 \\
\cline { 2 - 3 } & $\begin{array}{l}\text { Administración local: consejos } \\
\text { insulares (Islas Baleares) }\end{array}$ & 3 \\
\cline { 2 - 3 } & $\begin{array}{l}\text { Administración local: cabildos } \\
\text { insulares (Islas Canarias) }\end{array}$ & 7 \\
\hline Municipio & Administración local (ayuntamientos) & 1112 \\
\hline
\end{tabular}

Fuente: elaboración propia

Los autores ya demostraban en un reciente trabajo (Chain Navarro, Muñoz Cañavate \& Salido Martínez, 2008) cómo la Administración electrónica no es un objetivo estratégico para los estudios de Biblioteconomía y Documentación en España, por más que las posibilidades laborales, académicas y de investigación sean potencialmente mayores que las de los campos clásicos como las bibliotecas o los archivos. La propia gestión de los websites de las Administraciones Públicas encabeza uno de los sectores con más proyección, una vez que la Ley recientemente aprobada en España de Acceso Electrónico de los Ciudadanos a los Servicios Públicos, obliga a todas las Administraciones Públicas a ofrecer de manera telemática los servicios que ya vienen ofreciendo a ciudadanos y empresas de manera tradicional.

Así, en España, las relaciones entre los ciudadanos y las Administraciones no han sido ni un objetivo académico ni de investigación para los estudios universitarios de Biblioteconomía y Documentación, salvo excepciones.

\section{Comunicación y publicidad}

El último de los perfiles que estos autores quieren dar a conocer es el del denominado planner en la comunicación publicitaria (Marcos Recio, Sánchez Vigil y Villegas Tovar, 2007).

El mundo de los medios de comunicación es, sin duda, otro de los yacimientos de empleo más importantes que tienen los titulados en Documentación, aunque últimamente comience a cuestionarse la existencia de los centros de documentación de los medios debido al proceso de digitalización de los mismos, la facilidad de acceso a los datos, y al casi convencimiento de algunos de que el documentalista en los medios es una figura superflua.

En este último apartado se trata otra figura distinta que ha comenzado a tener una cierta importancia en las empresas de publicidad. El Diccionario de la RAE define publicidad como "la divulgación de noticias o anuncios de carácter comercial para atraer posibles compradores, espectadores, usuarios, etc.". Este proceso requiere de un conjunto 
de medios y de un conocimiento del entorno que permita diseñar una campaña. La necesidad de informes y estudios de todo tipo sobre los consumidores, sobre el propio cliente que encarga la campańa, o sobre la competencia de los productos de las campańas encargadas, requiere de este tipo de profesional.

Marcos Recio, et al. (2007) nos dicen que un planner debe realizar un trabajo de investigación sobre lo que buscan y desean los consumidores, debe plantear la estrategia que ayude a los creativos en su trabajo, debe ofrecer datos para la planificación, y como tal puede tener cabida en los departamentos de cuentas, de planificación y creatividad. La siguiente tabla nos muestra el número de empresas que bajo la denominación publicidad en el código CNAE existen en España. Si bien muchas no tienen asalariados o son empresas con una o dos personas, siguen existiendo miles de ellas en las que el volumen de las mismas sí permitiría vislumbrar la potenciación de este perfil en los estudios de Información y Documentación a través de enseñanzas ligadas, por ejemplo, a la inteligencia competitiva y la información empresarial.

Tabla 5 - Empresas de publicidad según código CNAE (actividad principal publicidad) en España 2008.

\begin{tabular}{|l|c|}
\hline Sin asalariados & 17.240 \\
\hline De 1 a 2 asalariados & 8.021 \\
\hline De 3 a 5 asalariados & 2.676 \\
\hline De 6 a 9 asalariados & 1.211 \\
\hline De 10 a 19 asalariados & 1.086 \\
\hline De 20 a 49 asalariados & 232 \\
\hline De 50 a 99 asalariados & 93 \\
\hline De 100 a 199 asalariados & 77 \\
\hline asalariados & 53 \\
\hline De 500 a 999 asalariados & 16 \\
\hline Más de 1000 asalariados & 10 \\
\hline Total & 30.715 \\
\hline
\end{tabular}

Fuente: DIRCE

\section{Conclusión}

Creemos que existen, y que van apareciendo, nuevas y variadas posibilidades de trabajo para los documentalistas. A la vez, también existen distintas barreras que no dejan ver este amplio mercado, aunque creemos que la más grave es la falta de visión por parte de los formadores y de los futuros profesionales. En un mercado donde la competencia es la clave del éxito, un titulado que sepa lo que quiere, lo que es capaz de hacer, y hacia dónde se quiere dirigir, tiene muchas más posibilidades de encontrar un trabajo acorde con su formación universitaria que otro que confía en las subvenciones estatales o en las salidas profesionales tradicionales. Las tres posibilidades de inserción en el mercado laboral que se han presentado aquí son algunas de las que se pueden abrir en el mercado para los gestores de información, tanto en la empresa privada como en la Administración Pública. 


\section{Referências bibliográficas}

Audunson, R. (2005). LIS and the Creation of a European Educational Space. Journal of Librarianship and Information Science, 37 (4), 171-174.

Chaín Navarro, C., Muñoz Cañavate, A. y Salido Martínez, V. (2008). LIS education and web services in the public sector: the case of Spain. Libri, 58, 246-256.

Delgado López-Cozar, E. (2007). El ocaso de las enseñanzas universitarias de Documentación en España. ThikEPI http://www.thinkepi.net.

García Marco, F.J. (2008). El movimiento iSchools: posicionando los estudios de Biblioteconomía y Documentación en la era de la información [17/10/2008] http://www.thinkepi.net/.

Marcos Recio J.C., Sánchez Vigil J.M. y Villegas Tovar, R. (2007). Los retos del documentalista/ planner en la comunicación publicitaria. En X Jornadas Españolas de Documentación. Santiago de Compostela, mayo, 363-371.

Martin-Moreno C, Garcia-Zorita C, Lascurain-Sanchez ML, Sanz-Casado E. (2005). Curricular design and labour market demand: Comparing three Carlos III University of Madrid curricula. Libri, 55, 2-3, 122-130.

Michavila, F. y Calvo, B. (1998). La Universidad española hoy. Madrid: Síntesis: 81-82.

Moscoso Castro, P. (2000). Tecnologías y Documentación: 25 años de desencuentros en la enseñanza de la Documentación. Actas I Congreso Universitario de Ciencias de la Documentación, pp. 87-100.

Muñoz Cańavate, A. (2007). La enseñanza de la información para la empresa en la universidad española. Reflexiones para una orientación académica y profesional de la disciplina. Documentación de las Ciencias de la Información, 30, 253-274.

Muńoz Cañavate, A. y Hípola Ruiz, P. (2008). An approach to information studies for the business world in spanish universities. Education for Information, 26 (3-4), 191-201.

Sanz Casado, E. et al. (2001). Las disciplinas científicas como encrucijada de saberes: el caso de los estudios de Biblioteconomía y Documentación en las Universidades espańolas. Revista General de Información y Documentación, 1 (11), 167-181.

Weeech, T.L. y Konieczny, A.M. (2007). Alternative careers for graduates of LIS schools. Journal of Librarianship and Information Science, 39 (2), 67-78. 